\title{
der junge zahnarzt
}

editorial

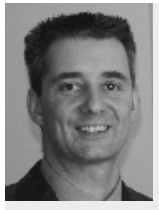

Dr. Carsten Hünecke //

ist allgemeinzahnärztlich in Magdeburg tätig und stellvertretender Vorsitzender des Landesverbands SachsenAnhalt im Freien Verband Deutscher Zahnärzte (FVDZ).

\section{Liebe Kolleginnen und Kollegen,}

„Meine Praxis - Einrichten und Gestalten!" - das Titelthema dieser Ausgabe hat es in sich. Geht es doch nicht nur um die richtige Ergonomie, das Umsetzen gesetzlicher Vorgaben, die richtigen Einheiten sowie die notwendigen Geräte. Das ist manchmal gar nicht so schwer, denn eigene Erfahrungen aus der Assistenzzeit und der Geldbeutel machen die Entscheidung möglicherweise leichter. Doch die Frage der "weichen Faktoren“ einer Praxis, die für unsere Patienten oft sehr hoch im Kurs stehen, ist da schon schwieriger: Welchen wichtigen ersten Eindruck vermittelt das Ambiente? Welche Botschaft möchte ich mit der Einrichtung versenden? Modern, zeitlos, klassisch, cool, wohnlich? Nicht zuletzt sagt die Ausstattung etwas über mich aus. So sollte die Einrichtung mir natürlich gefallen, schließlich verbringt man die nächsten 10-20 Jahre viel Zeit in der Praxis. Aber sie sollte auch den Patienten ansprechen, interessieren, eventuell beruhigen. Einrichtungsexperten, Farbspezialisten und Praxisplaner sind möglicherweise ganz hilfreich, am Ende sollten sie jedoch nur eines sein Berater. Oder warum nicht das gesamte Team mit einbinden? Die Identifikation mit der Praxis und der Motivationsschub sind nicht unerheblich für das Betriebsklima und das spürt der Patient genau. Wie dem auch sei, entscheiden muss man wohl allein, doch das mit einem möglichst guten Gefühl im Bauch.

Haben Sie vielleicht schon eigene Erfahrungen, dann schreiben Sie ruhig, die Redaktion und der Beirat freuen sich immer über Post.

In diesem Sinne viel Spaß bei Lesen, Ihr Carsten Hünecke

Dr. Christopher Köttgen, Mainz

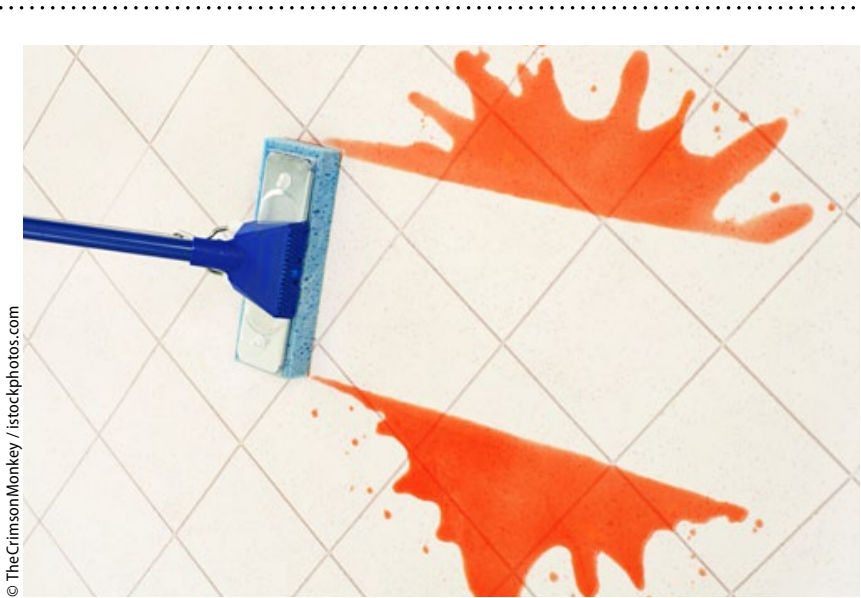

\section{fortbildung}

14 Validierung von Reinigungs- Desinfektions- und Sterilisationsverfahren

Die richtige Reinigung von Gerät und Praxis ist Pflicht und gesetzlich verankert. Lesen Sie hier, was wichtig ist.

\section{PD Dr. Lutz Jatzwauk // Dresden}

Mit diesem CME-Beitrag können zwei Fortbildungspunkte nach den Leitsätzen der Bundeszahnärztekammer zur zahnärztlichen Fortbildung einschließlich der Punktebewertung von BZÄK/ DGZMK erworben werden.

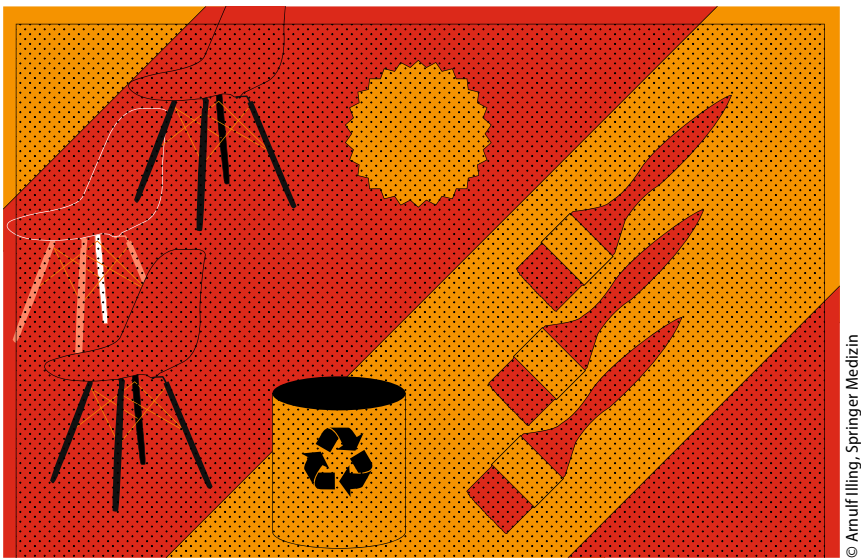

\section{titelthema}

34 Meine Praxis - Einrichten und Gestalten

Die Möglichkeiten scheinen unendlich, ebenso wie die Anforderungen an eine neue Praxis. Doch worauf kommt es wirklich an bei der ersten eigenen Praxis?

Dagmar Möbius // Berlin 


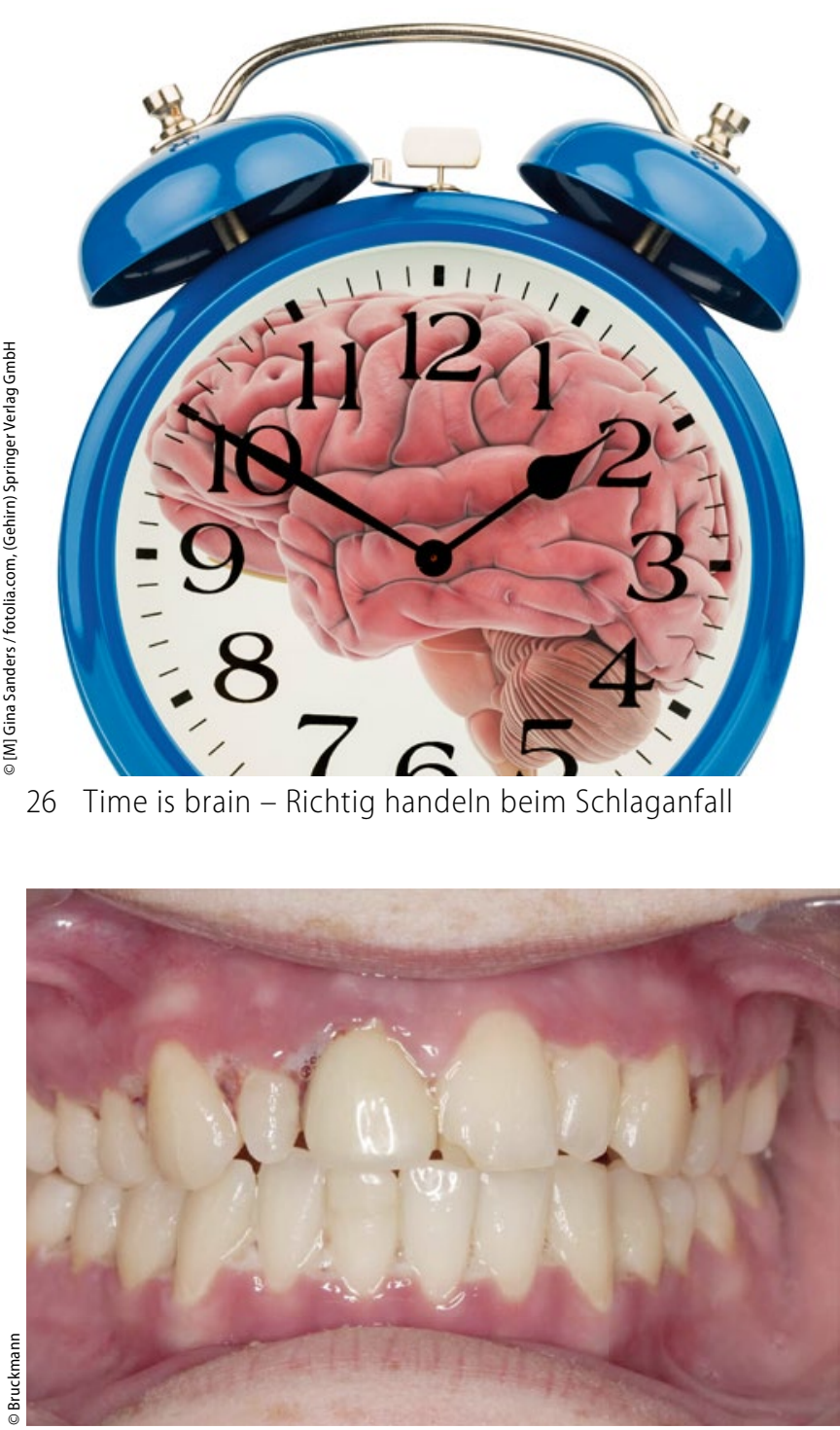

10 Wie rückt man solch einer nekrotisierenden Gingivitis am besten zu Leibe?

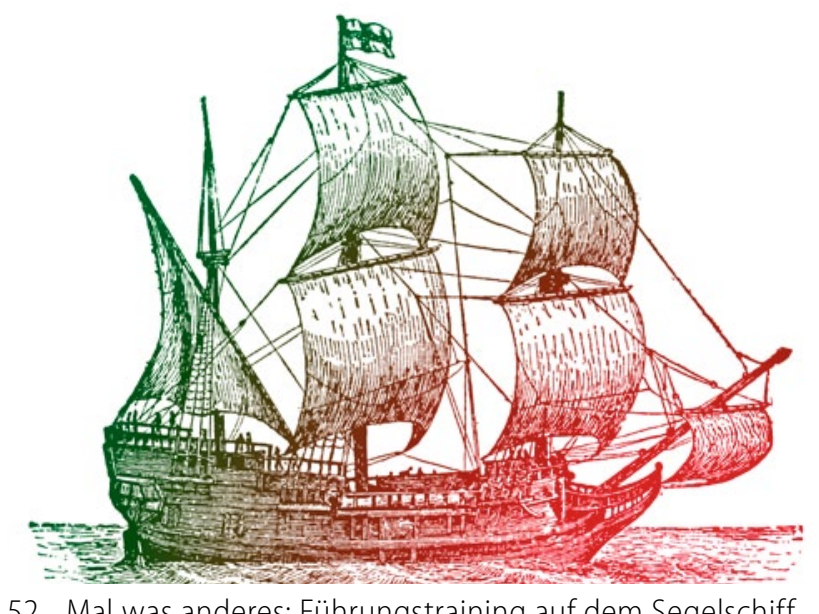

\section{einstieg}

$4 \quad$ Was bedeutet für Sie Nervenkitzel?

Gute Frage - gute Antwort

\section{medizin}

6 Regeneration des Pulpagewebes

Für Sie gelesen und kommentiert

10 Karius und Baktus in die Flucht schlagen!

Wann sollte man zur chemischen Keule greifen?

26 Der Schlaganfall: Time is brain!

Richtig handeln beim Notfall in der Zahnarztpraxis

29 Hätten Sie's gewusst?

Hygienequiz

\section{fortbildung}

30 Update Restaurative und Ästhetische Zahnheilkunde Gibt es Neues und Nützliches für die tägliche Praxis?

32 Chirurgische Behandlung tiefer Parodontaltaschen Schritt für Schritt erklärt

\section{praxis}

48 Wenn Zahnärzte kooperieren

Was ist erlaubt, was ist verboten?

50 Sorgen Sie vor, damit die Pflicht nicht das Genick bricht Alles Wichtige zur Haftpflichtversicherung

52 So führen Sie richtig!

Was man von alten Kapitänen alles lernen kann

54 IDS 2013 - eine Rückschau

Nicht nur die Fotoaktion von Springer war ein Renner

56 Erst anstellen lassen oder gleich allein durchstarten? Tipps für die Existenzgründung

01 editorial

58 mitteilungen fvdz und dgzmk

64 dentalforum

65 marktplatz

68 ausstieg, impressum 